\title{
Comparison of the Oral Steroids, Macrolides and Combination Therapy in Nasal Polyposis Patients
}

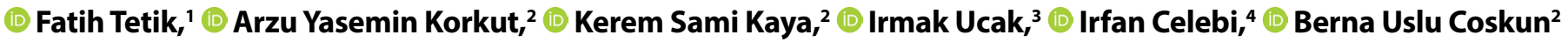 \\ 'Department of Otorhinolaryngology Head and Neck Surgery, Gaziosmanpasa Taksim Training and Research Hospital, Istanbul, Turkey \\ 2Department of Otorhinolaryngology Head and Neck Surgery, Sisli Hamidiye Etfal Training and Research Hospital, Istanbul, Turkey \\ ${ }^{3}$ Department of Otorhinolaryngology Head and Neck Surgery, Istanbul Sultan Abdulhamid Han Training and Research Hospital, \\ Istanbul, Turkey \\ ${ }^{4}$ Department of Radiology, Vehbi Koc Foundation American Hospital, Istanbul, Turkey
}

\begin{abstract}
Objectives: In this study, our aim was to compare oral steroid therapy with macrolide therapy and with oral steroid + macrolide (combine) therapy in patients with nasal polyposis (NP).

Methods: All patients were treated with nasal steroid therapy for eight weeks and divided randomly into three groups as follows: Oral steroid group, oral macrolide group and combine group. All patients underwent endoscopic staging, radiological grading, odour testing and completed the sino-nasal outcome test-22 (SNOT-22) questionnaire before and after treatment.

Results: Significant improvement was observed in all parameters after treatment in all three groups. All parameters were significantly better in the combined group than in the macrolide group. Comparison of the oral steroid group and macrolide group revealed significantly better radiological grading and odour test changes for the oral steroid group, but no statistically significant differences existed according to endoscopic staging and SNOT-22. The post-treatment SNOT-22 score was significantly better in the combined group than in the steroid group. A comparison of the combined and steroid groups showed better results for the combined group for all parameters, but the differences were not significant.

Conclusion: All treatment protocols were effective and the successful use of macrolide indicates its potential as an alternative in patients with contraindications to oral steroid treatment. The combined treatment may demonstrate significantly better results than steroid treatment alone if larger studies with more patients are performed.

Keywords: Chronic rhinosinusitis; macrolide; nasal polyposis; nasal steroid; oral steroid.

Please cite this article as "Tetik F, Korkut AY, Kaya KS, Ucak I, Celebi I, Uslu Coskun B. Comparison of the Oral Steroids, Macrolides and Combination Therapy in Nasal Polyposis Patients. Med Bull Sisli Etfal Hosp 2020;54(2):211-217".
\end{abstract}

$\mathrm{N}$ asal polyps (NP) are benign and are characterised by mucosal inflammation and expansion into the lumen of the nasal cavity. They are typically pale grey protrusions and are induced by multifactorial causes. The prevalence in the general population ranges between 1 and $4 \%$, and they mostly affect adult individuals. ${ }^{[1]}$ These polyps have been known since ancient times, and yet the pathogenesis and treatment of NP remain to be fully elucidated. In recent years, NP has been considered to represent a sub-group of chronic sinusitis. ${ }^{[2]}$ The most important factors in the development of nasal polyps are viewed as chronic inflammation and mucosal oedema. Other factors that may play a

Address for correspondence: Fatih Tetik, MD. Gaziosmanpasa Taksim Egitim ve Arastirma Hastanesi, Kulak Burun Bogaz ve Bas Boyun Cerrahisi Anabilim Dali, Istanbul, Turkey

Phone: +90 5059279426 E-mail: fatihtetik@windowslive.com

Submitted Date: July 19, 2018 Accepted Date: July 20, 2018 Available Online Date: June 12, 2020

${ }^{\circ}$ Copyright 2020 by The Medical Bulletin of Sisli Etfal Hospital - Available online at www.sislietfaltip.org

OPEN ACCESS This is an open access article under the CC BY-NC license (http://creativecommons.org/licenses/by-nc/4.0/). 
role include allergy, fungal and bacterial infections, and the biofilm formed. As a result of these, various chemical mediators (such as interleukin-5, tumour necrosis factor, nitric oxide), hypoxia due to osteomeatal obstruction, cyclooxygenase metabolism and some bacterial superantigens are present. ${ }^{[1]}$ Consideration of the Samter triad, cystic fibrosis and after-treatment recurrence indicates a genetic basis as well. ${ }^{[3]}$

Patients with NP have complaints, such as nasal occlusion, diminished sense of smell, hyponasal speech, fullness felt on the forehead, face, and cheeks, headache, nasal discharge and snoring. ${ }^{[4]}$ Nasal occlusion is the most significant complaint. A detailed history, endoscopic examination, laboratory tests, radiological evaluation, allergy testing and histopathological evaluation can be used to confirm the NP diagnosis. ${ }^{[5]}$

The purpose of NP treatment is to eliminate the polyps and the symptoms of rhinitis, restore nasal respiration and sense of smell, and prevent recurrences. Treatment of patients with NP is long-term and needs close follow-up. Medical or surgical treatment can be applied. Regardless of the treatment option, however, recurrences are quite frequent.

The treatment of NP can include the use of steroids, antibiotics, saline nasal spray, mucolytics, topical/systemic decongestants, topical anticholinergics, anti-leukotrienes or receptor blockers, and antihistamines, ${ }^{[4]}$ but steroids are the most effective drugs known for NP treatment. Steroids have a multitude of effects, including inhibition of cytokine synthesis, reduction of the number of eosinophils and activated eosinophils, anti-oedema effects and reduction of transudation. ${ }^{[6]}$ Specifically, macrolide antibiotics are thought to be effective, with an anti-inflammatory effect observed against chronic inflammation when used for a long duration. ${ }^{[7]}$

The present study aims to compare a low-dose long-term macrolide treatment with a combined oral steroid and macrolide treatment and with oral steroid treatment alone in patients with NP who experienced frequent recurrences despite all treatments and for whom a definitive treatment protocol could not be established.

\section{Methods}

This study started with the approval of code 1026 by the Institutional Ethics Committee of Clinical Investigations. This study was randomized and prospective.

\section{Patients}

Patients were included in this study if they were admitted to the ENT clinic of our hospital with NP diagnosed by en- doscopic and computed tomography (CT) examination, they were older than 18 years, and they provided detailed informed consent.

Exclusion criteria were patients with inverted papilloma or antrochoanal polyp with a unilateral polyp, advanced septum deviation that prevented endoscopic examination, a medical or surgical treatment for NP within the last three months and systemic diseases that constituted a contraindication for the treatment (uncontrolled diabetes, uncontrolled hypertension, chronic renal failure or glaucoma).

Patients included in this study were questioned in detail by the surgeon about when the complaints started, whether they suffered from asthma or allergic rhinitis or other additional diseases, and their surgical history related to NP.

\section{Endoscopic Staging}

The patients underwent an ear, nose and throat examination and then the endoscopic examination was performed (without a nasal decongestant) with a 0-degree rigid nasal endoscope with the patient in a sitting position. Both nasal cavities were assessed separately and scored according to the chronic rhinosinusitis staging system described in the guidelines for the European position paper on rhinosinusitis and nasal polyps 2012 (EPOS 2012). ${ }^{[8]}$ The polyp sizes were evaluated from 0 to 3 , with 0 being no polyp and 3 being polyps completely obstructing the nasal passage. The findings of oedema and discharge, as well as polyp sizes, were scored between 0 and 2 according to their severity. Values in both nasal passages were collected, and the total score between 0 and 14 of all patients was recorded.

\section{Radiological Staging}

The CT findings of the patients were evaluated according to the Lund-Mackay scoring system. ${ }^{[9]}$ This scoring system evaluates the occlusion of the osteomeatal complex and five major sinuses. ${ }^{[9]}$ The values on both sides were collected and the total scores were determined between 0 and 24 .

\section{Quality of Life Index}

The quality of life of the patients was evaluated by having the patients fill out the SNOT 22 questionnaire, which was translated into Turkish and validated and determined to be appropriate by Hanci et al. ${ }^{[10]}$ The SNOT-22 questionnaire consists of 22 questions and/or symptoms (no complaints= 0 , very severe complaints $=5$ ) answered by the patients. Average scores between 0 and 5 were found for the questionnaire.

\section{Odor Test}

The Connecticut Chemosensory Clinical Research Centre 
Test (CCCRC) was conducted as described previously elsewhere. ${ }^{[11,12]}$ The CCCRC test is composed of $n$-the butanol odour threshold test and odour identification test. Olfactory tests were scored out of 7 ( 0 : worst, 7: best olfaction) and the mean score was calculated as the total CCCRC test score. $^{[12]}$

\section{Treatments}

Patients were divided randomly in three groups and appropriate treatments were started according to their groups. All patients received $200 \mu \mathrm{g}$ mometasone furoate nasal spray twice a day for eight weeks.

The first group received oral steroid treatment in addition to nasal steroid spray.

The second group received antibiotic treatment in addition to nasal steroid spray.

The third group received both oral steroids and antibiotic therapy in addition to nasal steroid spray.

Prednisolone treatment was started by oral administration of tablets ( $1 \mathrm{mg} / \mathrm{kg} /$ day) as steroid therapy and the amount was decreased by $10 \mathrm{mg}$ every 2 days. The number of tablets was divided equally and administered daily in three doses. Groups receiving oral steroid treatment were administered $30 \mathrm{mg}$ lansoprazole tablets once daily for the duration of oral steroids, and an appropriate diet was recommended. As an antibiotic treatment, 500 mg clarithromycin tablets were given once a day for eight weeks.

When the 8-week treatment was completed, SNOT 22 questionnaires, endoscopic staging scores after endoscopic examination, odour tests and CT and radiological staging evaluation of the need for treatment and additional treatment were performed again.

\section{Statistical Methods}

Descriptive statistics included the mean value, standard deviation, median, minimum and highest frequency and rate values. The distribution of the variables was measured by the Kolmogorov-Smirnov test. The Mann-Whitney $\mathrm{U}$ test was used for quantitative analysis. The Wilcoxon test was used for the analysis of recurrent measurements. The Chisquare test was used for the analysis of qualitative data. The SPSS 22.0 program was used for statistical analysis. The sample size is calculated 66 at the alfa-significance level of 0.05 , predicting inter-group and intragroup medium effect differences are significant.

\section{Results}

A total of 67 patients was included in this study: 45 (67\%) male and 22 (33\%) female. The mean age of the patients was $44.5 \pm 14.0$ (18-74) years. The age of the patients, duration of disease, rate of surgery and asthma rate did not differ among the three groups ( $p>0.05)$. The number of male patients was significantly lower in the macrolide group than in the oral steroid group $(p<0.05)$. The gender distribution in the combined group did not differ significantly from those of the macrolide and oral steroid groups ( $p>0.05$ ) (Table 1).

No significant difference was noted between the groups when the endoscopic staging scores before treatment were compared in all three groups ( $p>0.05$ ). Endoscopic staging after treatment showed a significant decrease compared to the before treatment in all three groups $(p<0.05)$. Endoscopic staging scores after treatment was significantly higher in the macrolide group than in the combined group ( $p>0.05$ ). After treatment, endoscopic staging scores in the oral steroid group did not differ significantly from the scores of the

Table 1. General characteristics of the groups

\begin{tabular}{|c|c|c|c|c|c|c|c|c|c|c|}
\hline & \multicolumn{3}{|c|}{ Oral Steroid } & \multicolumn{3}{|c|}{ Macrolide } & \multicolumn{3}{|c|}{ Combination } & \multirow[b]{2}{*}{$\mathbf{p}$} \\
\hline & \multicolumn{2}{|c|}{ Avg. $\pm s, ~ s . / n-\%$} & Med. & \multicolumn{2}{|c|}{ Avg. $\pm s, ~ s . / n-\%$} & Med. & \multicolumn{2}{|c|}{ Avg. $\pm s, ~ s . / n-\%$} & Med. & \\
\hline Age & \multicolumn{2}{|c|}{$43.0 \pm 14.1$} & 40 & \multicolumn{2}{|c|}{$44.7 \pm 17.1$} & 42 & \multicolumn{2}{|c|}{$45.7 \pm 10.0$} & 47 & 0.360 \\
\hline Female & 4 & $18 \%$ & & 12 & $52 \%$ & & 6 & $27 \%$ & & \multirow[t]{2}{*}{0.042} \\
\hline Male & 18 & $82 \%$ & & 11 & $48 \%$ & & 16 & $73 \%$ & & \\
\hline No & 17 & $77 \%$ & & 15 & $65 \%$ & & 14 & $64 \%$ & & 0.565 \\
\hline Yes & 5 & $23 \%$ & & 8 & $35 \%$ & & 8 & $36 \%$ & & \\
\hline \multicolumn{11}{|l|}{ Asthma } \\
\hline No & 17 & $77 \%$ & & 19 & $83 \%$ & & 19 & $86 \%$ & & 0.732 \\
\hline Yes & 5 & $23 \%$ & & 4 & $17 \%$ & & 3 & $14 \%$ & & \\
\hline
\end{tabular}

Mann-Whitney $\mathrm{U}$ test/Chi-square test. 
macrolide and combination groups $(\mathrm{p}<0.05)$ (Table 2$)$.

Although after-treatment endoscopic staging scores decreased in all groups, the decrease in endoscopic staging scores was significantly higher in the combined group than in the macrolide group $(p<0.05)$. The after-treatment decrease in endoscopic staging scores in the oral steroid group did not differ significantly from the scores in the macrolide and combination groups ( $p>0.05$ ).

No significant difference was noted between the groups when the radiological grading values before treatment were compared in all three groups $(p>0.05)$. Radiologic grading scores after treatment in all three groups showed a significant decrease when compared to the before treatment scores $(p<0.05)$. The radiologic grading score after treatment was significantly higher in the macrolide group than in the oral steroid and combination groups $(p<0.05)$. After treatment, the radiological staging score in the oral steroids and combined groups did not differ significantly ( $>0.05$ ) (Table 3).

The after-treatment decrease radiological grade was significantly smaller in the macrolide group $(p<0.05)$ than in the oral steroid and combination groups. No significant difference was noted in the radiological grade score decrease after oral steroid and combination therapy.

No significant difference was noted among the groups when SNOT-22 values were compared before treatment in all three groups ( $p>0.05)$. The after-treatment SNOT-22 values in all three groups showed a significant decrease compared to the values before treatment $(p<0.05)$. The after-treatment SNOT-22 value was significantly lower in the combined group than in the oral steroid and macrolide groups $(p<0.05)$. The mean SNOT-22 value after oral steroid and macrolide treatment did not differ significantly ( $p>0.05$ ) (Table 4).

The after-treatment decrease in the SNOT-22 value was significantly larger in the combined group than in the macrolide group $(p<0.05)$. The decrease in SNOT-22 values after treatment in the oral steroid group did not differ significantly from values in the macrolide and combination groups ( $p>0.05$ ).

No significant difference was found between the groups when before and after-treatment odour test values were compared in all three groups ( $p>0.05)$. The after-treatment odour test results in oral steroid and combined groups showed a significant increase $(p<0.05)$ compared to the before treatment values $(p<0.05)$ (Table 5).

The after-treatment increase in the odour test results in the macrolide group was significantly smaller than the increase in the oral steroid and combination groups $(p<0.05)$. The after-treatment increase at the odour test results in oral steroids and combined groups did not differ significantly ( $p>0.05$ ).

Table 2. Endoscopic staging

\begin{tabular}{|c|c|c|c|c|c|c|c|}
\hline & \multicolumn{2}{|c|}{ Oral Steroid } & \multicolumn{2}{|c|}{ Macrolide } & \multicolumn{2}{|c|}{ Combination } & \multirow[b]{2}{*}{$\mathbf{p}$} \\
\hline & Avg. $\pm s, s$. & Med. & Avg. $\pm s, s$. & Med. & Avg. $\pm s, s$. & Med. & \\
\hline \multicolumn{8}{|l|}{ Endoscopic Staging } \\
\hline Before Treatment & $9.9 \pm 1.9$ & 10 & $10.0 \pm 1.9$ & 10 & $9.8 \pm 2.0$ & 10 & 0.939 \\
\hline After Treatment & $6.7 \pm 2.6$ & 7 & $7.1 \pm 1.9$ & 8 & $5.5 \pm 2.7$ & 5 & 0.048 \\
\hline BT/AT Change & $3.2 \pm 2.5$ & 3 & $2.9 \pm 2.1$ & 4 & $4.2 \pm 2.2$ & 4 & 0.047 \\
\hline Change $p$ & \multicolumn{2}{|c|}{0.000} & \multicolumn{2}{|c|}{0.000} & \multicolumn{2}{|c|}{0.000} & \\
\hline
\end{tabular}

Mann-Whitney U test/Wilcoxon test.

Table 3. Radiological staging

\begin{tabular}{|c|c|c|c|c|c|c|c|}
\hline & \multicolumn{2}{|c|}{ Oral Steroid } & \multicolumn{2}{|c|}{ Macrolide } & \multicolumn{2}{|c|}{ Combination } & \multirow[t]{2}{*}{$\mathbf{p}$} \\
\hline & Avg. $\pm s$, s. & Med. & Avg. $\pm s$, s. & Med. & Avg. $\pm s$, s. & Med. & \\
\hline \multicolumn{8}{|l|}{ Radiological } \\
\hline \multicolumn{8}{|l|}{ Staging } \\
\hline Before Treatment & $18.1 \pm 4.3$ & 19 & $17.7 \pm 4.0$ & 18 & $18.3 \pm 4.5$ & 19 & 0.833 \\
\hline After Treatment & $12.4 \pm 4.3$ & 13 & $15.2 \pm 5.2$ & 14 & $12.7 \pm 4.7$ & 11 & 0.024 \\
\hline BT/AT Change & $5.7 \pm 3.8$ & 5 & $2.5 \pm 4.2$ & 4 & $5.6 \pm 2.9$ & 6 & 0.025 \\
\hline Change $p$ & \multicolumn{2}{|c|}{0.000} & \multicolumn{2}{|c|}{0.022} & \multicolumn{2}{|c|}{0.000} & \\
\hline
\end{tabular}


Table 4. SNOT-22

\begin{tabular}{|c|c|c|c|c|c|c|c|}
\hline & \multicolumn{2}{|c|}{ Oral Steroid } & \multicolumn{2}{|c|}{ Macrolide } & \multicolumn{2}{|c|}{ Combination } & \multirow[t]{2}{*}{$\mathbf{p}$} \\
\hline & Avg. $\pm s, s$. & Med. & Avg. $\pm s$, s. & Med. & Avg. $\pm s, \mathrm{~s}$. & Med. & \\
\hline \multicolumn{8}{|l|}{ SNOT-22 } \\
\hline After Treatment & $1.9 \pm 0.7$ & 2 & $2.1 \pm 0.6$ & 2 & $1.4 \pm 0.7$ & 1 & 0.004 \\
\hline BT/AT Change & $0.9 \pm 0.5$ & 1 & $0.6 \pm 0.7$ & 1 & $1.2 \pm 0.9$ & 1 & 0.026 \\
\hline
\end{tabular}

Mann-Whitney U test/Wilcoxon test.

Table 5. Odor test

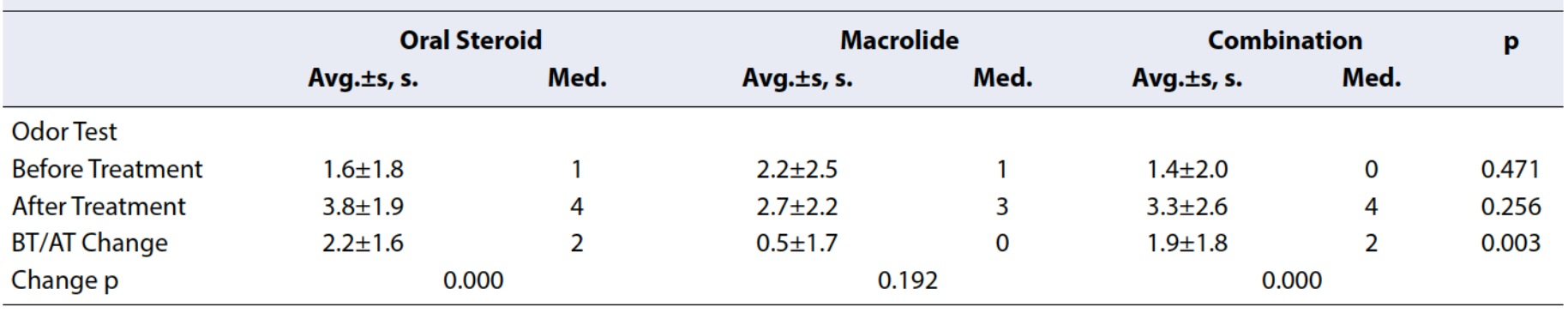

Mann-Whitney $\mathrm{U}$ test/Wilcoxon test.

\section{Discussion}

Despite its effectiveness as a medical treatment option for patients with NP, steroid therapy has limitations, such as contraindication in some patients and the inability to use the oral form long term or at frequent intervals. Patients with NP can be offered different medical treatment options, such as mucolytics, topical/systemic decongestants, topical anticholinergics, anti-leukotrienes or receptor blockers, antihistamines or saline nasal sprays ${ }^{[4]}$ However, none of these treatment options has achieved a high recommendation as NP treatments according to the results obtained in blood-based studies. ${ }^{[8]}$

Antibiotic treatment has not been considered an important alternative to steroid treatment in patients with NP, who have been treated as a subgroup of chronic rhinosinusitis in recent years. Nevertheless, this idea has been strengthened by the suggestion of the involvement of a regulatory effect of endotoxin-producing staphylococci in NP development. Schalek et al. ${ }^{[13]}$ conducted a placebo-controlled study in which 23 patients who were serologically positive for enterotoxin-producing Staphylococcus aureus strains and scheduled for endoscopic sinus surgery were randomised to receive a 3-week oral anti-staphylococcal antibiotic treatment (quinolone, amoxicillin/clavulanate or co-trimoxazole) or a placebo. Both groups were evaluated preoperatively at three and six months with endoscopic polyp scores and SNOT-22. The group using antibiotics showed better responses, but the differences did not reach the level of statistical significance.

Our review of the literature indicated that macrolide antibiotics have an anti-inflammatory effect on chronic inflammation in patients with NP when these antibiotics are used for a prolonged period. ${ }^{[7]}$ Yamada et al. ${ }^{[14]}$ treated 20 patients suffering from chronic rhinosinusitis and nasal polyps with clarithromycin (400 mg daily) for three months. In the group in which polyp sizes decreased, the interleukin-8 level decreased and their values had been significantly higher before macrolide treatment when compared to the group in which polyp sizes did not show any change. In a small study of twelve illnesses, a single daily dose of roxithromycin $(150 \mathrm{mg})$ decreased the interleukin-8 level and improved pneumatisation as determined by $\mathrm{CT}$. ${ }^{[15]}$

To our knowledge, there is not any study in the literature that have yet compared different antibiotic types for patients with NP. However, clarithromycin is preferred in most studies for long-term use. ${ }^{[14,16,17]}$ Anti-inflammatory effects were observed following the administration of clarithromycin at doses of $500 \mathrm{mg}$ and below for eight weeks or more. ${ }^{[13]}$ In our study, we used a single dose of a $500 \mathrm{mg}$ clarithromycin tablet daily for eight weeks as an antibiotic. Luo et al. ${ }^{[18]}$ administered a single dose of $250 \mathrm{mg}$ clarithromycin daily for 12 weeks in 50 patients with chronic sinusitis (33 patients with chronic sinusitis without polyps and 17 pa- 
tients with polyps). Comparison of the patient VAS scores, SNOT-20 scores and endoscopic and radiological grading scores before and after treatment revealed significant improvement in all findings in both groups, but the improvement in the patients who had chronic sinusitis with polyps was more prominent.

In our study, patients treated with macrolides showed significant improvement in endoscopic and radiological staging, SNOT-22 score and odour test after treatment. However, no definitive conclusion can be drawn regarding an antibiotic effect since all patients were administered nasal steroids and there was no control group.

Our examination of the literature did not reveal any studies comparing oral steroids with macrolide antibiotics and their combinations in patients with NP, only a comparison of oral steroids with doxycycline treatment for 20 days. ${ }^{[19]}$ Van Zele et al. ${ }^{[19]}$ conducted a placebo-controlled trial to compare a 20-day administration of doxycycline (200 mg first, followed by $100 \mathrm{mg}$ ) and a 3-week treatment with methylprednisolone ( 1 week $32 \mathrm{mg}, 1$ week $16 \mathrm{mg}$ and 1 week $8 \mathrm{mg}$ ) with the placebo. Inflammatory markers in the blood and nasal secretions were examined, polyp size was measured and the symptoms were recorded. Methylprednisolone had a short but dramatic effect on polyp size and symptoms. During the 12-week study period, doxycycline also had a small but significant effect on the polyp size compared to the placebo. Doxycycline had a significant effect on postnasal efflux, but it did not change the other symptoms. Examination of the nasal secretions revealed that doxycycline reduced myeloperoxidase (MPO) and eosinophilic cationic protein (ECP), as well as metallomatrix protein-9 (MMP-9). However, no quality of life measures was made, so it is not possible to determine whether doxycycline had an effect on the quality of life in this study group. In our study, comparison of the oral steroid group and macrolide group revealed a significantly better radiological grading and odour test response in the oral steroid group than in the macrolide group in terms of BT/AT changes. However, no significant difference was found between the two groups in terms of endoscopic staging and SNOT-22 BT/TS changes.

Kuran et al. ${ }^{[20]}$ added a treatment of clarithromycin $500 \mathrm{mg}$ twice daily for three weeks to a group of patients with nasal polyps who had received systemic steroids, nasal steroids, and antihistamine treatment and did not significantly improve after steroid treatment. Symptomatic improvement was observed in $80 \%$ of the patients and radiological improvement in $40 \%$ of the patients with antibiotic treatment. In our study, the combined treatment resulted in a significant improvement in endoscopic and radiological progression, SNOT-22 score and odour test. A comparison of the combined group with the macrolide group revealed that all parameters were significantly better in the combined group than in the macrolide group in terms of post-treatment values. Comparison of these two groups concerning BT/AT changes again showed significantly better results in all parameters in the combined group than in the macrolide group.

A comparison of the combined group with the steroid group, by contrast, revealed no significant differences between the two groups concerning post-treatment endoscopic staging, radiological staging and odour test results. However, the post-treatment SNOT-22 score was significantly better in the combined group than in the steroid group. Comparison of the two groups in terms of BT/AT changes indicated that the results were in favour of the combined group for all parameters, but the differences between the two groups were not statistically significant.

\section{Conclusion}

In conclusion, we observed that all the protocols used in our study were effective for the treatment of the NP. The improvement in the group given the combined treatment was significantly better than the macrolide group in all parameters. Significant differences were found between the steroid group and the macrolide group concerning radiological grading and improvement in the odour test. However, the differences between the two groups in terms of endoscopic staging and SNOT-22 healing were not statistically significant. No significant difference in improvement was found between the combined group and the steroid group. Taken together, these results suggest that the use of antibiotics of the macrolide type may represent an alternative for the treatment of NP in those patients with contraindications for oral steroid therapy. Further studies with large samples could reveal that the combined treatment alone will give significantly better results than steroid treatment.

\section{Disclosures}

Ethics Committee Approval: This study started with approval of code 1026 by Şişli Hamidiye Etfal Training and Research Hospital Ethics Committee of Clinical Investigations.

Peer-review: Externally peer-reviewed.

Conflict of Interest: None declared.

Authorship Contributions: Concept - F.T., A.Y.K.; Design - F.T., I.U.; Supervision - F.T., K.S.K.; Materials - F.T., K.S.K.; Data collection \&/or processing - F.T., I.C..; Analysis and/or interpretation - F.T., K.S.K., I.U.; Literature search - F.T., K.S.K.; Writing - F.T., K.S.K.; Critical review - A.Y.K., B.U.C. 


\section{References}

1. Andrews $A E$, Bryson JM, Rowe-Jones JM. Site of origin of nasal polyps: relevance to pathogenesis and management. Rhinology 2005;43:180-4.

2. Sabirov A, Hamilton RG, Jacobs JB, Hillman DE, Lebowitz RA, Watts JD. Role of local immunoglobulin E specific for Alternaria alternata in the pathogenesis of nasal polyposis. Laryngoscope 2008;118:4-9. [CrossRef]

3. Newton JR, Ah-See KW. A review of nasal polyposis. Ther Clin Risk Manag 2008;4:507-12. [CrossRef]

4. Sarıoglu T, Bayar N, Kanbur B, Gündüz M. Treatment methods in recurrent nasal polyps. Drug and Treatment Journal 1993;6:12-4.

5. Erbek SS, Erbek S, Budakoğlu I, Cakmak O. The relationship between smoking and nasal polyposis. Kulak Burun Bogaz Ihtis Derg 2008;18:216-20.

6. Stammberger H. Rhinoscopic surgery. In: Settipane GA, Lund VJ, Bernstein JM, Tos M, editor. Nasal polyps: epidemiology, pathogenesis and treatment. Rhode Island: Ocean Side Pub; 1997. p. 7-15.

7. Bachert C, Watelet JB, Gevaert P, Van Cauwenberge P. Pharmacological management of nasal polyposis. Drugs 2005;65:1537-52.

8. Fokkens WJ, Lund VJ, Mullol J, Bachert C, Alobid I, Baroody F, et al. EPOS 2012: European position paper on rhinosinusitis and nasal polyps 2012. A summary for otorhinolaryngologists. Rhinology 2012;50:1-12. [CrossRef]

9. Lund VJ, Mackay IS. Staging in rhinosinusitus. Rhinology 1993;31:183-4.

10. Hancı D, Altun H, Şahin E, Altıntoprak N, Cingi C. Turkish translation, cross-cultural adaptation and validation of the Sino Nasal Outcome Test (SNOT)-22. ENT Updates 2015;5:51-7. [CrossRef]

11. Kobayashi M, Reiter ER, DiNardo LJ, Costanzo RM. A new clinical olfactory function test: cross-cultural influence. Arch Otolaryngol Head Neck Surg 2007;133:331-6. [CrossRef]
12. Veyseller B, Ozucer B, Karaaltin AB, Yildirim Y, Degirmenci N, Aksoy $F$, et al. Connecticut (CCCRC) Olfactory Test: Normative Values in 426 Healthy Volunteers. Indian J Otolaryngol Head Neck Surg 2014;66:31-4. [CrossRef]

13. Schalek $P$, Petrás $P$, Klement V, Hahn A. Short-term antibiotics treatment in patients with nasal polyps and enterotoxins producing Staphylococcus aureus strains. Eur Arch Otorhinolaryngol 2009;266:1909-13. [CrossRef]

14. Yamada T, Fujieda S, Mori S, Yamamoto H, Saito H. Macrolide treatment decreased the size of nasal polyps and IL-8 levels in nasal lavage. Am J Rhinol 2000;14:143-8. [CrossRef]

15. Suzuki H, Shimomura A, Ikeda K, Oshima T, Takasaka T. Effects of long-term low-dose macrolide administration on neutrophil recruitment and IL-8 in the nasal discharge of chronic sinusitis patients. Tohoku J Exp Med 1997;182:115-24. [CrossRef]

16. Varvyanskaya A, Lopatin A. Efficacy of long-term low-dose macrolide therapy in preventing early recurrence of nasal polyps after endoscopic sinus surgery. Int Forum Allergy Rhinol 2014;4:53341. [CrossRef]

17. Perić A, Baletić N, Milojević M, Sotirović J, Živić L, Perić AV, et al. Effects of Preoperative Clarithromycin Administration in Patients with Nasal Polyposis. West Indian Med J 2014;63:721-7. [CrossRef]

18. Luo Q, Deng J, Xu R, Zuo K, Li H, Shi J. Clinical effect of clarithromycin therapy in patients with chronic rhinosinusitis. [Article in Chinese]. Zhonghua Er Bi Yan Hou Tou Jing Wai Ke Za Zhi 2014;49:103-8.

19. Van Zele T, Gevaert P, Holtappels G, Beule A, Wormald PJ, Mayr S, et al. Oral steroids and doxycycline: two different approaches to treat nasal polyps. J Allergy Clin Immunol 2010;125:1069-76.

20. Kuran G, Kocatürk S, Kurukahvecioğlu S, Erkam U. The efficacy of combined medical treatment (antihistaminics, steroids, and macrolide antibiotics) in patients with nasal polyposis: preliminary results. Kulak Burun Bogaz Ihtis Derg 2002;9:352-7. 